\title{
Association between Neutrophil to Lymphocyte Ratio and Inflammatory Markers in Hemodialysis Patients
}

\author{
Hafez Ahmed Abd El-Hafeez ${ }^{1}$, Ahmed Mohammed Al-Alashkar ${ }^{1}$, Nagah Mohamed Abo \\ Mohamed $^{2}$, Mohammed Ibrahim Khalil Zoheir ${ }^{1}$
}

${ }^{1}$ Department of Internal Medicine, Faculty of Medicine, Al-Azhar University

${ }^{2}$ Department of Clinical Pathology, Faculty of Medicine, Al-Azhar University

*Corresponding author: Mohammed Ibrahim Khalil Zoheir, Mobile: (+20) 01558336585, E-Mail: dr_Zohair@ yahoo.com

\begin{abstract}
Background: End stage renal disease patients on regular hemodialysis patients have higher rate of mortality and morbidity compared to the general population.

Objective: This study aimed to study the association between neutrophil-to-lymphocytic ratio and other inflammatory markers (IL-6, high sensitive CRP, PLR, etc) in hemodialysis patients.

Patients and methods: This study was designed to assess inflammation in hemodialysis patients and study the association between high sensitive CRP (hs-CRP) and the other inflammatory markers in those patients. Initially, forty two patients with end stage renal disease (ESRD), on regular hemodialysis in dialysis unit, Internal Medicine Department, AL-Hussien Hospital, Faculty of Medicine, Al-Azhar University-Egypt.

Results: We calculated the cut off point for IL- 6 that best indicates the presence of inflammation in hemodialysis patients, we found that it was value more than $30 \mathrm{ng} / \mathrm{l}$ with sensitivity 96.6 , specificity 69.2\%, with AUC: 00.77 and P $<0.004$. Moreover, we calculated the cutoff point for PLR that is consistent with presence of inflammation in hemodialysis patients, we found that it was more than 180.2 with sensitivity $82.2 \%$, specificity $92.3 \%$ with AUC .087 and p value equals 0.0001 . In addition to calculating the cut off point for NLR that best indicates the presence of inflammation, we found that it was $\geq 1.6$ with sensitivity $93.1 \%$, specificity $92.3 \%$, with AUC: 0.92 and $\mathrm{P}<0.001$.

Conclusion: We can assess cardiovascular condition of our hemodialysis patients along with their morbidity and mortality with measuring these inflammatory markers which are nonexpensive and has high sensitivity and specificity.
\end{abstract}

Keywords: Neutrophil to Lymphocyte Ratio, Hemodialysis Patients, ESRD, CVD

\section{Introduction}

Both cardiovascular diseases and infection are linked to inflammation and ESKD has recently been considered a state of chronic inflammation, which is the cornerstone of pathogenesis of atherosclerosis, is increased in ESRD patients compared to normal population. It is thought that early detection of inflammation might improve the quality of the life of those patients and decrease rate of morbidity and mortality ${ }^{(\mathbf{1})}$.

Patients on RHD have increased level of inflammatory mediators including $\mathrm{C}$ - reactive protein, tumour necrosis factor and IL6, as it plays major role in malnutrition, inflammation and atherosclerosis as well as overall mortality rate in these patients (2). Leukocyte are considered among the classic inflammatory markers due to their role in pathogenesis of atherosclerosis and its complications by mediating several biochemichal pathways ${ }^{(2)}$.
Several studies have revealed that elevated neutrophil count was strongly associated with malnutrition and inflammation and that decreased lymphocyte count had a weaker association. Increased neutrophils and decreased lymphocyte count were also independent predictor of mortality in hemodialysis patients ${ }^{(3)}$. Recently, neutrophil to- lymphocyte ratio is considered a novel cheap and available indicator, which reflect the extent of inflammation and atherosclerosis and predicts the clinical outcome and estimate survival in cardiac and non-cardiac including ESRD $^{(4)}$. The neutrophil to lymphocyte ratio is obtained by dividing the absolute neutrophil count by the absolute lymphocyte count. It is a marker of poor prognosis in several disorders like malignancies, chronic kidney disease and myocardial function (3). Based on that, the present study was designed to evaluate the NLR 
compared with hs-CRP along with IL6 in ESRD patients on regular hemodialysis.

\section{Aim of the work}

This study aimed to study the association between neutrophil-to-lymphocytic ratio and other inflammatory markers (IL-6, high sensitive CRP, PLR, etc) in hemodialysis patients.

\section{Patient and methods}

This study was designed to assess inflammation in hemodialysis patients and study the association between hs-CRP and the other inflammatory markers in those patients. Initially, forty two patients with end stage renal disease (ESRD), on regular hemodialysis in dialysis unit, Internal Medicine Department, AL-Hussien Hospital, Faculty of Medicine, AlAzhar University-Egypt, were recruited to participate in our study

Ethical approval and written informed consent:

An approval of the study was obtained from Al- Azhar University Academic and Ethical Committee. Every patient signed an informed written consent for acceptance of the operation.

\section{-Inclusion criteria:}

-Patients undergoing HD for more than three months who will agree to be included in this study.

\section{Exclusion criteria:}

- We are going to exclude patients who have

- Inflammatory state due to infection,

- Autoimmune diseases,

- Older than 75 year-old,

- Current malignancy or history of malignancy,

- Immunosuppressive therapy.

\section{Study design:}

We divided our patients into two groups above and below hs-CRP10 mg/dl, then we studied the correlation between Hs-CRP, NLR, PLR, IL6 and other predictors of inflammation in the group of CRP $>10 \mathrm{mg} / \mathrm{dl}$. After that we detected the cut off values for them.

\section{All subjects in this study were subjected to the following:}

1-Full history including age, weight, height ,BMI, history of high blood pressure ,diabetes ,peripheral vascular disease along with cerebrovascular disease.

\section{2- Laboratory investigations:}

Venous blood samples will be drawn from all subjects after an overnight fasting period. Sampling were particularly performed in a morning of midweek dialysis session prior to heparinization in HD patients. Then, we measured:

- Serum albumin, ferritin, mean platelet volume (MPV), high sensitive CRP and interleukin-6.

- Calcium

- Phosphorus

The white blood cell differentiation will be detected as part of $\mathrm{CBC}$, then we calculate neutrophil to lymphocyte ratio and platelet to lymphocyte ratio.

\section{Statistical analysis:}

Data were analyzed using Statistical Program for Social Science (SPSS) version 15.0. Quantitative data were expressed as mean \pm standard deviation (SD). Qualitative data were expressed as frequency and percentage. The following tests were done:

\section{Independent-samples t-test of significance} was used when comparing between two means.

ROC curve (Receiver Operating Characteristic Curve) was used to detect cutoff value, sensitivity, specificity, positive predictive value (PPV) and negative predictive value (NPV).

Sensitivity measures the proportion of actual positives that are correctly identified as such (e.g., the percentage of sick people who are correctly identified as having the condition).

Specificity measures the proportion of actual negatives that are correctly identified as such (e.g., the percentage of healthy people who are correctly identified as not having the condition).

Positive predictive value is the probability that subjects with a positive screening test truly have the disease.

Negative predictive value is the probability that subjects with a negative screening test truly don't have the disease. 
Pearson's correlation coefficient (r) test was used for correlating data.

\section{Probability (P-value)}

- P-value <0.05 was considered significant.

\section{Results}

Table (1): description of demographic data of studied patients

\begin{tabular}{|c|c|c|c|}
\hline Variables & & \multicolumn{2}{|c|}{ Studied patients $(\mathrm{N}=42)$} \\
\hline \multirow{2}{*}{ Age (years) } & Mean \pm SD & \multicolumn{2}{|c|}{$50.36 \pm 10.66$} \\
\hline & Min - Max & \multicolumn{2}{|c|}{$24-70$} \\
\hline \multirow{2}{*}{$\operatorname{Sex}(n, \%)$} & Male & 28 & $66.67 \%$ \\
\hline & Female & 14 & $33.33 \%$ \\
\hline \multirow{2}{*}{ Weight (kg) } & Mean \pm SD & \multicolumn{2}{|c|}{$74.05 \pm 13.66$} \\
\hline & Min - Max & \multicolumn{2}{|c|}{$53-120$} \\
\hline \multirow{2}{*}{ Height (m) } & Mean \pm SD & \multicolumn{2}{|c|}{$1.67 \pm 0.09$} \\
\hline & Min-Max & \multicolumn{2}{|c|}{$1.5-1.84$} \\
\hline \multirow{2}{*}{ BMI $\left(\mathbf{k g} / \mathbf{m}^{2}\right)$} & Mean \pm SD & \multicolumn{2}{|c|}{$26.92 \pm 5.35$} \\
\hline & Min - Max & \multicolumn{2}{|c|}{$19.11-46.89$} \\
\hline \multirow[b]{2}{*}{ Dialysis duration (years) } & Mean \pm SD & \multicolumn{2}{|c|}{$5.81 \pm 4.89$} \\
\hline & Min - Max & \multicolumn{2}{|c|}{$0.5-17$} \\
\hline \multirow{2}{*}{ DM $(\mathbf{n}, \%)$} & No & 34 & $80.95 \%$ \\
\hline & Yes & 8 & $19.05 \%$ \\
\hline \multirow{2}{*}{ HTN $(n, \%)$} & No & 10 & $23.81 \%$ \\
\hline & Yes & 32 & $76.19 \%$ \\
\hline \multirow{2}{*}{ Cerebrovascular Ds (n, \%) } & No & 39 & $92.86 \%$ \\
\hline & Yes & 3 & $7.14 \%$ \\
\hline \multirow{2}{*}{ Cardiovascular Ds (n, \%) } & No & 22 & $52.38 \%$ \\
\hline & Yes & 20 & $47.62 \%$ \\
\hline
\end{tabular}

This table shows description of demographic data of studied patients.

As regards age, the mean age of studied patients was $50.36 \pm 10.66$ years old with minimum age of 24 years and maximum age of 70 years. As regards sex, there were 28 males $(66.6 \%)$ and 14 females $(33.33 \%)$ in studied patients. As regards weight, the mean weight of studied patients was $74.05 \pm 13.66 \mathrm{~kg}$ with minimum weight of $53 \mathrm{~kg}$ and maximum weight of $120 \mathrm{~kg}$. As regards height, the mean height of studied patients was $1.67 \pm 0.09 \mathrm{~m}$ with minimum height of $1.5 \mathrm{~m}$ and maximum height of $1.84 \mathrm{~m}$. As regards BMI, the mean BMI of studied patients was $26.92 \pm 5.35 \mathrm{~kg} / \mathrm{m}^{2}$ with minimum BMI of $19.11 \mathrm{~kg} / \mathrm{m}^{2}$ and maximum BMI of $46.89 \mathrm{~kg} / \mathrm{m}^{2}$. As regards dialysis duration, the mean dialysis duration of
- P-value <0.001 was considered as highly significant.

- P-value > 0.05 was considered insignificant. studied patients was $5.81 \pm 4.89$ years with minimum duration of 0.5 year and maximum duration of 17 years. As regards DM, there were 34 non-diabetic $(80.95 \%)$ and 8 diabetic $(19.05 \%)$ in studied patients. As regards HTN, there were 10 non-hypertensive $(23.81 \%)$ and 32 hypertensive $(76.19 \%)$ in studied patients. As regards cerebrovascular diseases, there were 39 patients with no cerebrovascular diseases $(92.86 \%)$ and 3 patients with cerebrovascular diseases $(7.14 \%)$ in studied patients. As regards cardiovascular diseases, there were 22 patients with no cardiovascular diseases $(52.38 \%)$ and 20 patients with cardiovascular diseases $(47.62 \%)$ in studied patients as shown in table (1). 
Table (2): Correlation study between Hs-CRP, NLR, PLR \& IL-6 in patients with CRP > 10mg/dl group

\begin{tabular}{|l|c|c|c|c|c|c|c|c|}
\hline \multirow{2}{*}{} & \multicolumn{2}{|c|}{ Hs-CRP } & \multicolumn{2}{c|}{ NLR } & \multicolumn{2}{c|}{ PLR } & \multicolumn{2}{c|}{ IL-6 } \\
\cline { 2 - 10 } & r & P-value & r & P-value & r & P-value & r & P-value \\
\hline Hs-CRP & --- & --- & 0.65 & $<0.001 *$ & 0.54 & $0.002 * *$ & 0.45 & $0.013 * *$ \\
\hline NLR & 0.65 & $<0.001 *$ & --- & --- & 0.89 & $<0.001 *$ & 0.53 & $0.003 * *$ \\
\hline PLR & 0.54 & $0.002 * *$ & 0.89 & $<0.001 *$ & --- & --- & 0.54 & $0.002 * *$ \\
\hline IL-6 & 0.45 & $0.013 * *$ & 0.53 & $0.003 * *$ & 0.54 & $0.002 * *$ & --- & - \\
\hline
\end{tabular}

(r): Pearson correlation coefficient.

$*$ : p-value $<0.001$ is considered highly significant.

$* *$ : p-value $<0.05$ is considered significant.

This table showed:

- Highly statistical significant ( $\mathrm{p}$-value $<0.001$ ) positive correlation between hs-CRP vs NLR \& NLR vs PLR in patients with CRP $>10 \mathrm{mg} / \mathrm{dl}$ group.

- Statistically significant ( $\mathrm{p}$-value < 0.05 ) positive correlation between hs-CRP vs PLR, hs-CRP vs IL-6, NLR vs IL-6 \& PLR vs IL-6 in patients with CRP > $10 \mathrm{mg} / \mathrm{dl}$ group.

Table (3): Correlation study between Hs-CRP and other studied parameters in patients with CRP > $10 \mathrm{mg} / \mathrm{dl}$ group

\begin{tabular}{|l|c|c|l|c|c|}
\hline \multirow{2}{*}{\multicolumn{1}{|c|}{ Variables }} & \multicolumn{2}{|c|}{ Pearson Corr. } & \multirow{2}{*}{ Variables } & \multicolumn{2}{c|}{ Pearson Corr. } \\
\cline { 2 - 3 } \cline { 6 - 7 } & r & P-value & & r & P-value \\
\hline Hs-CRP vs T. Ca & 0.08 & 0.67 & Hs-CRP vs Hb & -0.55 & 0.009 \\
\hline Hs-CRP vs Ionized Ca & -0.22 & 0.24 & Hs-CRP vs NLR & $\mathbf{0 . 6 5}$ & $<\mathbf{0 . 0 0 1 *}$ \\
\hline Hs-CRP vs Ca x Ph & -0.3 & 0.1 & Hs-CRP vs PLR & $\mathbf{0 . 5 4}$ & $\mathbf{0 . 0 0 2 * *}$ \\
\hline Hs-CRP vs Ph & -0.35 & 0.056 & Hs-CRP vs MPV & -0.25 & 0.17 \\
\hline Hs-CRP vs T. Sat. & 0.2 & 0.27 & Hs-CRP vs IL-6 & $\mathbf{0 . 4 5}$ & $\mathbf{0 . 0 1 3 * *}$ \\
\hline Hs-CRP vs TIBC & -0.39 & 0.6 & Hs-CRP vs PTH & -0.04 & 0.83 \\
\hline Hs-CRP vs iron & 0.057 & 0.77 & Hs-CRP vs ALB & -0.65 & 0.007 \\
\hline Hs-CRP vs ferritin & $\mathbf{0 . 7 6}$ & $\mathbf{0 . 0 0 8}$ & & & \\
\hline
\end{tabular}

(r): Pearson correlation coefficient.

$*$ : p-value $<0.001$ is considered highly significant.

$* *$ : p-value $<0.05$ is considered significant.

This table showed:

- Highly statistical significant ( $\mathrm{p}$-value $<0.001$ ) positive correlation between hs-CRP vs NLR in patients with CRP > $10 \mathrm{mg} / \mathrm{dl}$ group. 
- Statistically significant ( $\mathrm{p}$-value < 0.05 ) positive correlation between hs-CRP vs PLR, hs-CRP vs IL-6 and hs-CRP vs Ferritin as well as statistically significant (p-value $<0.05$ ) negative correlation between hs-CRP vs ALB and hs-CRP vs $\mathrm{Hb}$ in patients with CRP $>10 \mathrm{mg} / \mathrm{dl}$ group.

- No statistical significant (p-value > 0.05) correlation between hs-CRP and other studied parameters in patients with CRP $>10 \mathrm{mg} / \mathrm{dl}$ group.

Table (4): Correlation study between NLR and other studied parameters in patients with CRP > $10 \mathrm{mg} / \mathrm{dl}$ group

\begin{tabular}{|l|c|c|l|c|c|}
\hline \multirow{2}{*}{\multicolumn{1}{|c|}{ Variables }} & \multicolumn{2}{|c|}{ Pearson Corr. } & \multirow{2}{*}{ Variables } & \multicolumn{2}{c|}{ Pearson Corr. } \\
\cline { 2 - 3 } \cline { 6 - 7 } & $\mathbf{r}$ & P-value & & $\mathbf{r}$ & P-value \\
\hline NLR vs T. Ca & -0.09 & 0.63 & NLR vs ferritin & $\mathbf{0 . 5 5}$ & $\mathbf{0 . 0 1 * *}$ \\
\hline NLR vs Ionized Ca & -0.035 & 0.059 & NLR vs Hb & $\mathbf{- 0 . 6 5}$ & $\mathbf{0 . 0 0 9 * *}$ \\
\hline NLR vs Ca x Ph & -0.15 & 0.41 & NLR vs PLR & 0.89 & $<\mathbf{0 . 0 0 1 *}$ \\
\hline NLR vs Ph & -0.15 & 0.42 & NLR vs MPV & -0.49 & $\mathbf{0 . 0 0 7 * *}$ \\
\hline NLR vs T. Sat. & 0.1 & 0.58 & NLR vs IL-6 & 0.53 & $\mathbf{0 . 0 0 3 * *}$ \\
\hline NLR vs TIBC & -0.27 & 0.15 & NLR vs PTH & 0.14 & 0.44 \\
\hline NLR vs iron & -0.02 & 0.9 & NLR vs ALB & $-\mathbf{0 . 7 7}$ & $\mathbf{0 . 0 0 3 * *}$ \\
\hline
\end{tabular}

(r): Pearson correlation coefficient.

*: p-value $<0.001$ is considered highly significant.

$* *$ : p-value $<0.05$ is considered significant.

This table showed:

- Highly statistical significant (p-value $<\mathbf{0 . 0 0 1 )}$ positive correlation between NLR vs PLR in patients with CRP $>10 \mathrm{mg} / \mathrm{dl}$ group.

- Statistically significant (p-value $<\mathbf{0 . 0 5}$ ) positive correlation between NLR vs IL-6 and NLR vs ferritin as well as statistically significant (p-value $<\mathbf{0 . 0 5}$ ) negative correlation between NLR vs MPV, NLR vs Hb and NLR vs ALB in patients with CRP > $10 \mathrm{mg} / \mathrm{dl}$ group.

- No statistical significant (p-value > 0.05) correlation between NLR and other studied parameters in patients with CRP $>10 \mathrm{mg} / \mathrm{dl}$ group.

Table (5): Correlation study between PLR and other studied parameters in patients with CRP > 10mg/dl group

\begin{tabular}{|c|c|c|c|c|c|}
\hline \multirow{2}{*}{ Variables } & \multicolumn{2}{|c|}{ Pearson Corr. } & \multirow{2}{*}{ Variables } & \multicolumn{2}{|c|}{ Pearson Corr. } \\
\hline & $\mathbf{r}$ & P-value & & $\mathbf{r}$ & P-value \\
\hline PLR vs T. Ca & -0.13 & 0.47 & PLR vs ferritin & 0.89 & $0.007 * *$ \\
\hline PLR vs Ionized Ca & -0.36 & 0.005 & PLR vs Hb & -0.67 & $0.008 * *$ \\
\hline PLR vs Ca x Ph & -0.27 & 0.14 & PLR vs NLR & 0.89 & $<0.001 *$ \\
\hline PLR vs Ph & -0.22 & 0.23 & PLR vs MPV & -0.49 & 0.7 \\
\hline
\end{tabular}




\begin{tabular}{|l|c|c|l|c|c|}
\hline PLR vs T. Sat. & 0.088 & 0.64 & PLR vs IL-6 & 0.54 & $\mathbf{0 . 0 0 2} * *$ \\
\hline PLR vs TIBC & -0.25 & 0.18 & PLR vs PTH & 0.26 & 0.16 \\
\hline PLR vs iron & 0.05 & 0.77 & PLR vs ALB & $\mathbf{- 0 . 7 2}$ & $\mathbf{0 . 0 0 1 * *}$ \\
\hline
\end{tabular}

(r): Pearson correlation coefficient.

*: p-value $<0.001$ is considered highly significant.

$* *$ : p-value $<0.05$ is considered significant.

This table showed

- Highly statistical significant (p-value $<\mathbf{0 . 0 0 1})$ Positive correlation between PLR vs NLR in patients with CRP $>10 \mathrm{mg} / \mathrm{dl}$ group.

- Statistically significant (p-value $<\mathbf{0 . 0 5}$ ) positive correlation between PLR vs IL-6 and PLR vs ferritin as well as statistically significant $(\mathbf{p}$-value $<\mathbf{0 . 0 5})$ negative correlation between PLR vs $\mathrm{Hb}$ and PLR vs ALB in patients with CRP $>10 \mathrm{mg} / \mathrm{dl}$ group.

- No statistical significant (p-value > 0.05) correlation between PLR and other studied parameters in patients with CRP $>10 \mathrm{mg} / \mathrm{dl}$ group.

Table (6): Correlation study between IL-6 and other studied parameters in patients with CRP > 10mg/dl group.

\begin{tabular}{|l|c|c|l|c|c|}
\hline \multirow{2}{*}{\multicolumn{1}{c|}{ Variables }} & \multicolumn{2}{c|}{ Pearson Corr. } & \multicolumn{2}{c|}{ Variables } & \multicolumn{2}{c|}{ Pearson Corr. } \\
\cline { 2 - 4 } & r & P-value & & r & P-value \\
\hline IL-6vs T. Ca & -0.04 & 0.8 & IL-6 vs ferritin & $\mathbf{0 . 8 8}$ & $\mathbf{0 . 0 0 7 * *}$ \\
\hline IL-6 vs Ionized Ca & -0.32 & 0.08 & IL-6 vs Hb & $\mathbf{0 . 2 4}$ & $\mathbf{0 . 0 3} * *$ \\
\hline IL-6 vs Ca x Ph & -0.008 & 0.9 & IL-6 vs NLR & $\mathbf{0 . 5 3}$ & $\mathbf{0 . 0 0 3} * *$ \\
\hline IL-6 vs Ph & -0.003 & 0.9 & IL-6 vs MPV & -0.31 & 0.1 \\
\hline IL-6 vs T. Sat. & 0.06 & 0.73 & IL-6 vs PLR & $\mathbf{0 . 5 4}$ & $\mathbf{0 . 0 0 2 * *}$ \\
\hline IL-6 vs TIBC & -0.35 & 0.06 & IL-6 vs PTH & 0.07 & 0.71 \\
\hline IL-6 vs iron & -0.04 & 0.8 & IL-6 vs ALB & $\mathbf{0 . 3 4}$ & $\mathbf{0 . 0 1} * *$ \\
\hline
\end{tabular}

(r): Pearson correlation coefficient.

$* *$ : p-value $<0.05$ is considered significant.

This table showed:

- Statistically significant (p-value $<\mathbf{0 . 0 5}$ ) positive correlation between NLR vs IL-6 and (IL-6 vs ferritin as well as statistically significant (p-value $<\mathbf{0 . 0 5}$ ) negative correlation between PLR vs IL-6, IL-6 vs $\mathrm{Hb}$ and IL-6 vs ALB in patients with CRP > $10 \mathrm{mg} / \mathrm{dl}$ group.

- No statistical significant (p-value > 0.05) correlation between IL-6 and other studied parameters in patients with CRP $>10 \mathrm{mg} / \mathrm{dl}$ group.

Table (7): Diagnostic performance of NLR to predict cases with inflammation

\begin{tabular}{|c|c|c|c|c|c|c|c|}
\hline Cut off & $\begin{array}{c}\text { Area under th } \\
\text { curve }\end{array}$ & Sensitivity & Specificity & PPV & NPV & p-value \\
\hline$>1.6$ & & 0.92 & $93.1 \%$ & $92.3 \%$ & $92.4 \%$ & $93.04 \%$ & $<0.001$ \\
\hline
\end{tabular}

PPV: positive predictive value.

NPV: negative predictive value.

Using roc curve, it was shown that NLR can be used to predict cases with inflammation at a cutoff level >1.6, with $93.1 \%$ sensitivity, $92.3 \%$ specificity, $92.4 \%$ PPV and $93.4 \%$ NPV.

Table (8): Diagnostic performance of IL-6 to predict cases with inflammation 
ejhm.journals.ekb.eg

\begin{tabular}{|c|c|c|c|c|c|r|}
\hline Cut off & $\begin{array}{c}\text { Area under the } \\
\text { curve }\end{array}$ & Sensitivity & Specificity & PPV & NPV & p-value \\
\hline$>30$ & 0.77 & $96.6 \%$ & $69.2 \%$ & $75.8 \%$ & $95.3 \%$ & 0.004 \\
\hline
\end{tabular}

PPV: positive predictive value.

NPV: negative predictive value.

Using roc curve, it was shown that IL-6 can be used to to predict cases with inflammation at a cutoff level > 30, with 96.6\% sensitivity, 69.2\% specificity, $75.8 \%$ PPV and 95.3\% NPV.

Table (9): Diagnostic performance of PLRto predict cases with inflammation

\begin{tabular}{|c|c|c|c|r|r|r|}
\hline Cut off & $\begin{array}{r}\text { Area under the } \\
\text { curve }\end{array}$ & Sensitivity & Specificity & PPV & NPV & p-value \\
\hline$>180.2$ & 0.87 & $82.8 \%$ & $92.3 \%$ & $91.5 \%$ & $84.3 \%$ & $\mathbf{0 . 0 0 0 1}$ \\
\hline
\end{tabular}

Using roc curve, it was shown that PLR can be used to predict cases with inflammation at a cutoff level >180.2, with $82.8 \%$ sensitivity, $92.3 \%$ specificity, $91.5 \%$ PPV and $84.3 \%$ NPV.

\section{Discussion}

This was a cross-sectional study designed to study the relation between hs-CRP as a marker of inflammation compared to NLR, IL-6 and PLR in ESRD patients on maintenance HD as well as studying the prevalence of subclinical inflammation those patients.

Sixty three $(69 \%)$ patients were found to have a high hs-CRP ( $>10 \mathrm{mg} / \mathrm{L})$ indicating presence of inflammation, despite absence of any overt signs and symptoms of inflammation. This goes in agreement with Korevaar et al. ${ }^{(8)}$ who reported that the prevalence of inflammation in HD patients varied between $35 \%$ and $65 \%$. Other results goes in favor with the chronic subinflammatory state present in ESRD, where Dai et al. ${ }^{(1)}$ concluded that dialysis-related factors such as use of catheters for vascular access, poor dialyzer membrane biocompatibility, dialysate contamination, exposure to endotoxins, and back-leak of dialysate across the dialysis membrane in hemodialysis (HD) may promote a persistent, low-grade inflammatory response. Besides, other comorbidities, kidney disease per se, life style factors, genetic predisposition and, in particular, the state of uremia is of major importance as a promoter of a persistent, low-grade inflammatory response in ESRD patients.

Our results showed a negative significant correlation between hs-CRP and albumin $(\mathrm{r}=-0.65, \mathrm{p}=0.007)$ along with hemoglobin $(\mathrm{r}=-0.55, \mathrm{p}=0.009)$. These results are consistent with the study of Ozcicek et al. ${ }^{(6)}$ for hemoglobin $(r=-0.66, p=0.001)$ whereas for albumin $(\mathrm{r}=-0.38, \mathrm{p}=<0.001)$. On the other side, our study revealed no statistical significance regarding Calcium $(\mathrm{p}=0.67)$, phosphorus $(\mathrm{p}=.056)$, TSAT $(\mathrm{p}=0.27)$ as well as iron $(\mathrm{p}=0.77)$, MPV $(\mathrm{p}=0.17)$ and PTH (p $=0.83$ ).

Further results showed positive correlation with significance between hs-CRP and PLR $(\mathrm{p}=0.002)$. These results are consistent with the study of Ahbap et al. ${ }^{(2)}$ where Both NLR and PLR were positively correlated with hs-CRP ( $\mathrm{r}$ $=0.333, \mathrm{p}=0.01$ and $\mathrm{r}=0.262, \mathrm{p}=0.001$, respectively).

Furthermore, we found a statistically significant positive correlation between NLR and hs-CRP $(\mathrm{r}=0.65, \mathrm{p}<0.001)$. These results are consistent with the studies of Pineault et al. ${ }^{(7)}$ who showed a positive correlation between hsCRP and NLR ( $\mathrm{r}=0.45, \mathrm{P}<.001)$, Ahbap et al. 
(2) who found same correlation (with $p<0.001$ ) and the study of Neuen et al. ${ }^{(8)}(r=0.24, p<$ 0.0023 ).

In addition, our results revealed a positive significant correlation between hs-CRP and IL$6 \mathrm{p}=0.13$. This study is consistent with Taheri et al. (9) who found a positive significant correlation between hs-CRP and IL-6 ( $<$ < $0.001)$.

In terms of independent predictors of inflammation in our study, it showed statistically significant in views to ferritin ( $\mathrm{p}=$ 0.0016), HB ( $p=0.027)$, IL-6 (0.002) and albumin $(\mathrm{p}=0.009)$, whereas, it revealed highly significance regarding NLR $(\mathrm{p}<0.001)$ and PLR ( $\mathrm{p}<0.001$ ). By using univariate and multiple variate analysis to test for independent predictors of hs -CRP levels as an indicator for inflammation, it was found that NLR can be used as an independent predictor of hs-CRP as an inflammatory marker, with statistically significant correlation $(\mathrm{p}<0.001)$.

To our knowledge, we are one of the few studies to determine a cutoff point for NLR as well as IL-6 and PLR as predictors of inflammation and to assess its sensitivity and specificity. We calculated the cut off point for IL-6 that best indicates the presence of inflammation in hemodialysis patients. We found that its value was more than $30 \mathrm{ng} / \mathrm{l}$ with sensitivity of 96.6 , specificity of $69.2 \%$ and AUC of 00.77 (P < 0.004). Moreover, we calculated the cutoff point for PLR that was consistent with presence of inflammation in hemodialysis patients. We found that it was more than 180.2 with sensitivity of $82.2 \%$, specificity of $92.3 \%$ and AUC of .087 ( $p$ value equals 0.0001). In addition to calculating the cut off point for NLR the best indicator for the presence of inflammation, we found that it was $\geq 1.6$ with sensitivity of $93.1 \%$, specificity of $92.3 \%$ and AUC of 0.92 ( $\mathrm{P}<0.001)$. In contrast to our results, Ahbap et al. ${ }^{(2)}$ found a cutoff point of 2.82 with sensitivity of $65.7 \%$ and specificity of $63.3 \%$.

As the American Heart Association (CDC/AHA) suggested use of hs-CRP cut points of low risk $(<1.0 \mathrm{mg} / \mathrm{L})$, average risk $(1.0-3.0 \mathrm{mg} / \mathrm{L})$ and high risk $(>3.0 \mathrm{mg} / \mathrm{L}) . \mathrm{In}$ this regard, Ahbap et al. ${ }^{(2)}$ data were compared in patients with hs-CRP levels of $\leq 3 \mathrm{mg} / \mathrm{L}$ vs. $>3 \mathrm{mg} / \mathrm{L}$ in the study, while our reference range that indicated inflammation was $\geq 8.2 \mathrm{mg} / \mathrm{L}$ (according to kits reference range), difference in reference ranges might explain the different cutoff values.

Other studies determined NLR as a marker and predictor of CVD mortality. Solak et al. ${ }^{(10)}$ and Abe et al. ${ }^{(11)}$ studies reported NLR > 3.76 and 3.72 to be significant and independent of CRP predictors of cardiovascular events in dialysisdependent patients, respectively. While An $\boldsymbol{e t}$ al. ${ }^{(12)}$, reported that NLR > 3.5 was associated with an increase in the risk of cardiovascular and all- cause mortality in peritoneal dialysis patients. Neuen et al. ${ }^{(8)}$, reported that NLR > 3.3 was associated with increased cardiovascular mortality in hemodialysis patients.

On further classification of the study group according to previously calculated cut off point for NLR, it was found that $69 \%$ of patients were considered positive for inflammation and $31 \%$ without inflammation.

\section{Conclusion}

According to this study, most of end stage renal disease patients on regular hemodialysis had established inflammation, which is a major risk for CVDS. Moreover, we studied the association between neutrophil to lymphocyte ratio and the other inflammatory markers in those patients. Moreover, we could detect cut off values of IL6, NLR and PLR in our patients which had high sensitivity and specificity compared to hs-CRP.

\section{Recommendations}

We recommend as a result of this study using simple tests like NLR, PLR and high sensitive CRP for the assessment of cardiovascular risk in hemodialysis patients beside the other traditional methods. Additionally, we detected cut off values for inflammation in hemodialysis patient.

\section{References}

1. Dai L, Golembiewska E, Lindholm B et al. (2017): End-Stage Renal Disease, Inflammation and Cardiovascular Outcomes. Contrib Nephrol., 191: 32-43.

2. Ahbap E, Sakaci T, Kara E et al. (2016): Neutrophil-to-lymphocyte ratio and platelet-tolymphocyte ratio in evaluation of inflammation in end-stage renal disease. Clinical Nephrology, 85 (4): 199-208. 
3. Yaprak M, Turan MN, Dayanan R et al. (2016): Platelet-to-lymphocyte ratio predicts mortality better than neutrophil-tolymphocyte ratio in hemodialysis patients,Int Urol Nephrol., 48: 1343.

4. Gürağaç A, Demirer $Z$ (2016): The neutrophil-to-lymphocyte ratio in clinical Practice. Can Urol Assoc J., 10 (3-4): 141.

5. Korevaar JC, Van Manen JG, Dekker FW et al. (2004): Effect of an increase in C-reactive protein level during a hemodialysis session on mortality. J Am Soc Nephrol., 15 (11): 2916-2922.

6. Ozcicek A, Ozcicek F, Yildiz G et al. (2017): Neutrophil-to-lymphocyte ratio as a possible indicator of epicardial adipose tissue in patients undergoing hemodialysis. Archives of Medical Science: AMS., 13 (1): 118-123.

7. Pineault $\mathrm{J}$, Lamarche $\mathrm{C}$, Bell $\mathrm{R}$ et al. (2017): Association of Neutrophil-toLymphocyte Ratio with Inflammation and Erythropoietin Resistance in Chronic Dialysis Patients. Canadian Journal of Kidney Health and Disease, 4: 1-7.
8. Neuen BL, Leather $\mathrm{N}$, Greenwood AM et al. (2016): Neutrophil-lymphocyte ratio predicts cardiovascular and all-cause mortality in hemodialysis patients. Renal Failure, 38 (1): 70-76.

9. Taheri S, Lin L, Austin D et al. (2004): Short sleep duration is associated with reduced leptin, elevated ghrelin, and increased body mass index. PLoS Med., 1: 62-6.

10. Solak Y, Yilmaz MI, Sonmez A et al. (2013): Neutrophil to lymphocyte ratio independently predicts cardiovascular events in patients with chronic kidney disease. Clin Exp Nephrol., 17: 532-540.

11. Abe T, Kato S, Tsuruta Y et al. (2014): Neutrophil/Lymphocyte ratio as a predictor of cardiovascular events in incident dialysis patients: A Japanese prospective cohort study. Clin Exp Nephrol., 19: 718-24.

12. An X, Mao HP, Wei $X$ et al. (2012): Elevated neutrophil to lymphocyte ratio predicts overall and cardiovascular mortality in maintenance peritoneal dialysis patients. Int Urol Nephrol., 44: 1521-1528. 